\title{
Las Competencias Parentales en Contextos de Riesgo Psicosocial
}

\section{Parental Competences in Psychosocial Risk Context}

\author{
M. ' José Rodrigo López \\ Universidad de La Laguna \\ Eduardo Cabrera Casimiro \\ Ayuntamiento de San Bartolomé de Tirajana
}

\author{
Juan Carlos Martín Quintana \\ Universidad de Las Palmas de Gran Canaria \\ M. ${ }^{a}$ Luisa Máiquez Chaves \\ Universidad de La Laguna
}

\begin{abstract}
Resumen. El análisis y la evaluación de las competencias parentales es una tarea central en los Servicios Sociales y concretamente en los Servicios especializados de atención a las familias en situación de riesgo psicosocial. Es imprescindible conocerlas y evaluarlas, entre otros motivos, para tomar medidas de apoyo a la unidad familiar o incluso para determinar si es necesario retirar a un menor de su familia. Paradójicamente, ésta ha sido un área de investigación poco explorada y ha ocupado también un lugar secundario en el campo de la protección de menores. Por ello, es importante clarificar qué se entiende por competencia parental, cuáles son las competencias parentales consideradas básicas para la educación saludable y positiva de los menores y poder contar con directrices claras para que los técnicos puedan llevar a cabo la evaluación de las mismas. El presente artículo pretende dar respuesta a estas cuestiones mencionadas.
\end{abstract}

Palabras claves: competencia parental, evaluación de competencias, familias en riesgo psicosocial.

Summary. The analysis and evaluation of parental competences is a central task for the Social Services and specifically for those services targeted at families living in psychosocial risk circumstances. The evaluation of parental competence can be used, among others motives, to take support measures to the family or even to determine if it is necessary to displace the children from his/her home. Despite of its importance, it is an area of sudy underexploited that also plays a secondary role in the field of child protection. For this reason, it is important to clarify what is meant by parental competence, which are the parental competences considered as basic for the healthy and positive parenting of the children, as well as to have clear guidelines to profesionals for carrying out the assessment of these competences. The current article tries to give answers to the questions mentioned.

Keywords: parental competence, competence assessment, families at psychosocial risk.

\section{Introducción}

Desde una perspectiva evolutivo-educativa la familia es un grupo humano que tiene como misión construir un escenario adecuado para el desarrollo

La correspondencia sobre este artículo puede dirigirse a la primera autora a la Facultad de Psicología de La Laguna. 38071 Tenerife (Spain).e-mail: mjrodri@ull.es de personas y apoyarlas en su proceso de aprendizaje (Rodrigo y Palacios, 1998). En concreto, la tarea educativa de los padres es bastante compleja ya que está encaminada a promover el desarrollo de los menores y, para ello, ésta debe desplegarse en diferentes niveles de actuación, desde el nivel estratégi-

Proyecto financiado por el Ministerio de Ciencia e Innovación (Proyecto TRA 2009-0145). Concedido a la primera autora. 
co de organización del escenario educativo hasta el nivel táctico de selección de actuaciones concretas (Máiquez, Rodrigo, Martín y Vermaes, 2001). Parece evidente que, para un óptimo desarrollo de dicha tarea, los padres necesiten disponer de competencias que les permitan proteger a los menores a su cargo y favorecer su desarrollo positivo.

Paradójicamente, son escasos los estudios que se han ocupado del análisis y la evaluación de las competencias parentales. Básicamente, dichos estudios se han llevado a cabo principalmente en dos ámbitos. Por un lado, en contextos judiciales en los que la evaluación de las competencias parentales es útil para la determinación, por ejemplo, de la custodia y/o separación de los menores de sus progenitores (Reder, Duncan y Lucey, 2003). Por otro, en contextos de riesgo psicosocial, para conocer las capacidades de los padres en la prevención del maltrato infantil y/o promover el desarrollo de programas de intervención con las familias vulnerables a este respecto (Azar y Cote, 2002).

Así pues, el análisis de las competencias parentales es crucial para que los servicios de protección de menores puedan evaluar mejor el ejercicio de la parentalidad en familias en riesgo y eventualmente tomar decisiones sobre la permanencia o no del menor en el hogar. De hecho, los modelos más recientes de protección de menores ya tienen como eje central la evaluación de las competencias parentales. Un ejemplo de ello, es el modelo para la evaluación de los menores y las familias en riesgo psicosocial desarrollado por el Departamento de Salud del Reino Unido (Department of Health, 2000). Las áreas evaluadas por este marco de referencia son las siguientes: a) la satisfacción de las necesidades evolutivas del menor (vg. salud, educación, desarrollo emocional); b) los factores ambientales y familiares que condicionan el ejercicio de la parentalidad (vg., empleo, vivienda, recursos comunitarios, historia familiar); y c) las competencias parentales. Las dimensiones de la capacidad parental evaluadas por este modelo son las siguientes: a) Cuidados básicos; b) Garantías de seguridad en la protección del menor; c) Calidez emocional; d) Estimulación del menor; e) Guía y límites al comportamiento del menor; y f) Estabilidad en sus vidas. Asimismo, es crucial contar con un buen análisis de las competen- cias parentales para elaborar programas de educación parental que adopten una visión optimizadora del ejercicio de la parentalidad en familias normalizadas o que promuevan, al menos, una parentalidad mínima para aquellas familias en riesgo psicosocial (Gordon, Bradley, Aue y Holman, 2006; Kalil, 2003; Tomison, 1999)

En este artículo nos ocuparemos en primer lugar, de definir el concepto de competencia parental; en segundo lugar, describiremos aquellas competencias necesarias para un buen ejercicio de la parentalidad $\mathrm{y}$, por último, propondremos algunas recomendaciones para evaluar dichas competencias y realizar un informe técnico sobre las mismas.

\section{El concepto de competencia parental}

La competencia es un concepto integrador que se refiere a la capacidad de las personas para generar y coordinar respuestas (afecto, cognición, comunicación y comportamiento) flexibles y adaptativas a corto y a largo plazo ante las demandas asociadas a la realización de sus tareas vitales y generar estrategias para aprovechar las oportunidades que les brindan los contextos de desarrollo (Masten y Curtis, 2000; Waters y Sroufe, 1983). Esta definición implica que la competencia es multidimensional, bidireccional, dinámica y contextual. Multidimensional porque implica el funcionamiento integrado de la cognición, el afecto y el comportamiento. Bidireccional porque sirve tanto para propiciar el ajuste personal y social a los contextos como para analizar lo que los contextos proporcionan a las personas en su desarrollo. Dinámica porque cambia a medida que el individuo se enfrenta a nuevos retos y tareas evolutivas que debe resolver, así como, a expectativas sociales que debe cumplir. Por último, el concepto de competencia es contextual en un doble sentido, porque las tareas evolutivas se practican en contextos vitales y porque tales contextos ofrecen oportunidades para nuevos aprendizajes y prácticas.

Siguiendo a Hawkins, Catalano y Miller (1992), las competencias requieren oportunidades para practicarlas, el aprendizaje de habilidades para poder utilizar las oportunidades que se le brindan y el reconocimiento social de la tarea vital bien hecha 
para seguir motivados a continuar y perfeccionar sus habilidades. Tanto las oportunidades, el entrenamiento de habilidades y el reconocimiento de haberlas adquirido se las brindan los contextos de desarro1lo, familia, escuela, iguales y ocio. Todo ello resulta crucial para un buen desarrollo de competencias.

Centrándonos en el tema de las competencias parentales, las definimos como aquel conjunto de capacidades que permiten a los padres afrontar de modo flexible y adaptativo la tarea vital de ser padres, de acuerdo con las necesidades evolutivas y educativas de los hijos e hijas y con los estándares considerados como aceptables por la sociedad, y aprovechando todas las oportunidades y apoyos que les brindan los sistemas de influencia de la familia para desplegar dichas capacidades (Rodrigo, Máiquez, Martín y Byrne, 2008).

Las competencias parentales son el resultado de un ajuste entre las condiciones psicosociales en las que vive la familia, el escenario educativo que los padres o cuidadores han construido para realizar su tarea vital y las características del menor (White, 2005). Por ejemplo, condiciones psicosociales como la monoparentalidad, el bajo nivel educativo, la precariedad económica y vivir en barrios violentos, entre otros factores, convierten la tarea de ser padre o madre en una tarea difícil. Sin embargo, si los padres cuentan con determinadas competencias podrían no sólo no comprometer el desarrollo de sus hijos sino incluso favorecer su resiliencia. Así, por ejemplo, aquellos padres que, a pesar de la adversidad, se centran en sus hijos y tienen expectativas positivas sobre su futuro, potencian más la resiliencia de los hijos que aquellos que cuentan con expectativas no realistas o que no tienen expectativas (Rodríguez, Camacho, Rodrigo, Martín y Máiquez, 2006)

En cuanto al escenario educativo, es evidente que es necesario analizar las concepciones y las prácticas educativas utilizadas por los padres en la crianza de los hijos. Al fin y al cabo, las prácticas educativas que los padres emplean para corregirle o el modo en que interactúan con el niño o el adolescente conforman el escenario de desarrollo del menor (Rodrigo et al. 2008). Los estudios sobre resiliencia parental nos ha mostrado cómo se pueden construir escenarios educativos adecuados en contextos de riesgo, sin que se de un impacto negativo sobre el desarrollo del menor (Kalil, 2003). Por ejemplo, se sabe que una supervisión parental más estricta facilita una mejor adaptación del menor en contextos con alto nivel de delincuencia (Cauce, Stewart, Rodríguez, Cochran y Ginzler, 2003).

Por último, las características del menor, es decir, su vulnerabilidad y su resiliencia, deben ser factores a considerar para determinar qué competencias parentales habría que potenciar en sus progenitores. Los menores, por ejemplo, con características particulares como la premadurez y/o bajo peso al nacer, la discapacidad física o psíquica, los problemas de conducta, la hiperactividad, los problemas de sueño, los problemas de control de esfínteres, los problemas de alimentación, o el temperamento difícil, entre otras características (Belsky y Jaffee, 2006; Díaz, Pérez, Martínez, Brito y Herrera, 2001; Simón, Correa, Rodrigo y Rodríguez, 1998) pueden hacer que la tarea de educarlos suponga ajustes y compensaciones que otros niños no necesitan. Por ello puede ser necesario potenciar en sus padres determinadas competencias que son cruciales para el cuidado y desarrollo positivo de estos niños.

No obstante, hay que recordar que, junto a estos rasgos de vulnerabilidad, debemos tener también en cuenta las características resilientes de los menores que tienen un ajuste personal y social mejor que el que cabría esperar teniendo en cuenta las condiciones adversas en que viven (Luthar, 2003). La mayor parte de los estudios coinciden en señalar las siguientes características: buena competencia social, inteligencia media o superior, temperamento fácil, locus de control interno, alta autoestima, sentido del humor, búsqueda de apoyo de "otros" positivos, capacidad para solucionar problemas, iniciativa y toma de decisiones, orientación al futuro, y entusiasmo y motivación por las cosas. Estos factores podrían paliar o aminorar los efectos negativos de los contextos de riesgo.

\section{Análisis de las competencias parentales}

Como se comentó anteriormente son pocos los estudios que analizan las competencias básicas que son necesarias para la tarea de ser padres y menos aun los que identifican el conjunto de competencias 
que habría que promover en los contextos de riesgo psicosocial en los que se encuentran determinadas familias.

En el ámbito de la investigación e intervención en situaciones de maltrato infantil, Sandra Azar y colaboradores (Azar y Cote, 2002) han desarrollado un modelo cognitivo conductual que considera que una parentalidad competente tiene que ver fundamentalmente con la capacidad de adaptación de los padres. Es decir, los padres necesitan ser los suficientemente flexibles para adaptarse positivamente a las circunstancias y necesidades de sus hijos que van cambiando con la edad. Esta autora ha delineado las capacidades requeridas para ejercer las funciones parentales aunque también afirma que dichas capacidades no difieren mucho de las necesarias para funcionar satisfactoriamente en las relaciones interpersonales en general (Azar y Weinzierl, 2005). Esta autora agrupa en cinco áreas las distintas competencias parentales:

1. Educativas (p.e. manejo del niño, cuidado físico, seguridad, expresión emocional).

2. Sociocognitivas (p.e. perspectivismo, expectativas adecuadas respecto a las capacidades infantiles, estilo de atribución positivo, autoeficacia).

3. Autocontrol (p.e control de impulsos, percepciones precisas, habilidades de autocontrol, asertividad).

4. Manejo del estrés (p.e auto-cuidado, relajación, capacidad para divertirse, mantenimiento del apoyo social, capacidad de afrontamiento, planificación).

5. Sociales (p.e. solución de problemas interpersonales, empatía, reconocimiento de las emociones).

Por otro lado y situado en un contexto de valoración judicial, Peter Reder y colaboradores (Reder y Lucey, 1995; Reder, Duncan y Lucey, 2003) sugieren que para el ejercicio de la parentalidad se necesita disponer de competencias relacionadas con los siguientes ámbitos: a) funcionamiento personal (resiliencia, agencia personal, reflexión sobre la propia conducta); b) competencias asociadas al rol parental propiamente dicho (cuidados físicos y emocionales, compromiso con la tarea parental, métodos disciplinarios, aceptación de la responsabilidad parental); y c) competencias asociadas a la relación con el niño (interés en las experiencias y bienestar del niño, empatía, reconocimiento de las necesidades del niño).

A partir de análisis de la bibliografía así como de nuestra experiencia en la formación de familias en riesgo psicosocial hemos estructurado las competencias parentales necesarias para la crianza de los hijos en cinco categorías de habilidades: educativas, agencia parental, autonomía y desarrollo personal, vida personal y organización doméstica (Cuadro 1).

Como se puede observar, las habilidades son de muy variado signo y deben considerarse relacionadas entre sí. Como cabría esperar las primeras son las habilidades educativas entre las que destacamos, por estar asociadas a la resiliencia familiar, las habilidades asociadas a la organización de actividades de ocio con toda la familia, o la educación en valores. Se encuentran también aquellas habilidades que permiten adecuar las pautas educativas al niño y realizar un continuo ajuste a sus peculiaridades: la observación, el perspectivismo, la sensibilidad y la reflexión (Azar y Cote, 2002).

Cuando hablamos de agencia parental estamos haciendo referencia a un conjunto de habilidades que reflejan el modo en que los padres perciben y viven su rol parental. Estas competencias permiten que los padres se sientan agentes protagonistas, activos, capaces y satisfechos en su rol parental (Máiquez, Rodrigo, Capote y Vermaes, 2000). También queda incluida aquí el reconocimiento y aceptación por parte de los padres de la importancia que ellos tienen en el bienestar del menor.

En cuanto a las habilidades de autonomía personal y búsqueda de apoyo social, aunque aparentemente contrapuestas forman, si se consigue un ajustado equilibrio, un conjunto de habilidades que se complementan entre sí. Esto se ve claramente en los programas de intervención con familias en riesgo psicosocial ya que si potenciamos el apoyo pero no el sentido de autonomía podemos promover la dependencia del técnico. Pero, por otro lado, si potenciamos la autonomía pero no la búsqueda y conservación de los apoyos podríamos sobrecargar los recursos de la familia y propiciar innecesariamente el estrés parental. 
Cuadro 1. Conjunto de habilidades requeridas para la tarea vital de ser padres (tomado de Rodrigo et al., 2008)

\section{HABILIDADES}

\begin{tabular}{l|l}
\hline Habilidades educativas & - Calidez y afecto en las relaciones y reconocimiento de los logros evolutivos alcanzados a la medida de
\end{tabular}
sus posibilidades.

- Control y supervisión del comportamiento del menor gracias a la comunicación y fomento de la confianza en sus buenas intenciones y capacidades y organización de actividades de ocio con toda la familia.

- Estimulación y apoyo al aprendizaje: fomento de la motivación, proporcionar ayuda contingente a las capacidades del menor, planificación de las actividades y tareas, orientación hacia el futuro e implicación en la educación formal (escuela).

- Actitud ética ante la vida y educación en valores.

- Adaptabilidad a las características del menor: capacidad de observación y flexibilidad para ajustarse a los cambios evolutivos, perspectivismo (capacidad de ponerse en el lugar del otro), autocorrección ante los errores, reflexión sobre la praxis educativa y flexibilidad para aplicar las pautas educativas.

- Autoeficacia parental: Percepción de las propias capacidades para llevar a cabo el rol de padres.

- Locus de control interno: Percepción de que se tiene control sobre sus vidas y capacidad de cambiar lo que ocurre a su alrededor que deba ser cambiado.

Habilidades de Agencia parental

- Acuerdo en la pareja: se acuerdan con la pareja los criterios educativos y los comportamientos a seguir con los hijos.

- Percepción ajustada del rol parental: Se tiene una idea realista de que la tarea de ser padres implica esfuerzo, tiempo y dedicación.

- Reconocimiento de la importancia de los progenitores en el bienestar del menor.

Habilidades de autonomía personal y búsqueda de apoyo social

- Implicación en la tarea educativa.

- Responsabilidad ante el bienestar del niño.

- Visión positiva del niño y de la familia.

- Buscar ayuda de personas significativas con el fin de complementar el rol parental en lugar de sustituirlo o devaluarlo.

- Identificar y utilizar los recursos para cubrir las necesidades como padres y como adultos.

- Búsqueda de ayuda de personas significativas y/o instituciones cuando tiene problemas personales y/o con los hijos.

- Confíanza y colaboración con los profesionales e instituciones que les quieren ofrecer apoyo y ayuda.

Habilidades para la vida personal

- Control de los impulsos.

- Asertividad.

- Autoestima

- Habilidades sociales.

- Estrategias de afrontamiento ante situaciones de estrés .

- Resolución de conflictos interpersonales.

- Capacidad para responder a múltiples tareas y retos.

- Planificación y proyecto de vida .

- Visión optimista y positiva de la vida y de los problemas y crisis.

Habilidades de organización doméstica
- Administración eficiente de la economía doméstica.

- Mantenimiento de la limpieza y orden de la casa.

- Higiene y el control de salud de los miembros de la familia.

- Preparación regular de comidas saludables.

- Arreglos y mantenimiento de la vivienda.
Las habilidades para la vida personal hacen referencia a aquellas habilidades que los padres y madres tienen que desarrollar para afrontar con más garantías de futuro su propia vida de adultos. Es bien conocido por los técnicos que trabajan con familias en riesgo psicosocial que estos padres, debido a varias condiciones como su propia historia personal de carencias afectivas, dificultad para establecer relaciones de intimidad con la pareja, falta de habilidades sociales, abandono prematuro de la escolaridad que compromete la entrada en el mundo laboral y la obtención estable de recursos económicos, escasas redes de apoyo social, etc., afrontan la tarea de ser padres demasiado pronto y con escasos recursos personales y sociales.

Por último, otra área importante que requiere de competencias específicas es la que tiene que ver con la organización doméstica. La preparación regular 
de comidas saludables, la administración de la economía doméstica, la limpieza y orden de la casa, así como el cuidado por la higiene y salud de todos los miembros de la familia son algunos de los aspectos incluidos en este tipo de habilidades que son cruciales cuando la familia está asediada por múltiples factores de riesgo.

\section{Recomendaciones para la evaluación de las competencias parentales}

La evaluación de las competencias parentales es una pieza clave en todo informe de valoración de familias con menores en situación de riesgo de desprotección. El esquema elaborado por Budd (2005) para la confección de informes en casos de protección de menores marca algunas directrices para llevar a cabo una adecuada evaluación de las competencias parentales.

En primer lugar, la evaluación de las competencias parentales debe contener información sobre los siguientes aspectos:

- La evaluación debe centrarse en las características de la parentalidad y las relaciones padreshijos y no tanto en la personalidad o funcionamiento cognitivo de los padres como adultos. Esto significa que, por ejemplo, no interesa tanto un diagnóstico clínico de trastorno de la personalidad del padre o la madre sino cómo este trastorno repercute en el bienestar del menor.

- Realizar un análisis funcional analizando las creencias y conocimientos evolutivo-educativos de los padres, sus expectativas sobre los logros del hijo y sobre sus metas educativas, asî como los comportamientos educativos y las habilidades parentales que despliegan en las actividades de la vida diaria.

- Buscar evidencia de una adecuación parental mínima o resiliente a las necesidades y características de los hijos, en lugar de buscar un estándar óptimo de parentalidad. Para ello hay que evaluar las características y necesidades del menor en función de su etapa evolutiva, ya que la valoración de si las capacidades parentales son suficientes o no puede variar en función de tales características y necesidades.
- Describir las fortalezas actuales de los padres y no sólo las debilidades en lo que se refiere a las relaciones padres-hijos y a la cobertura de las necesidades específicas de los hijos.

- Identificar aquellas condiciones contextuales (sociales, ambientales o históricas) que pudieran estar influyendo positiva o negativamente en la capacidad parental (vg., riesgos y apoyos) para educar a los hijos.

- La evaluación debería incluir un pronóstico así como recomendaciones sobre posibles intervenciones a realizar para fortalecer las competencias parentales, así como, los servicios y recursos que podrían ser de utilidad.

Respecto a la elaboración del informe sobre la evaluación realizada, debemos asegurarnos que se contestan aquellas cuestiones o dudas que pusieron en marcha el caso y se dejan claras las recomendaciones o decisiones a tomar a partir de estas respuestas. Además, su redacción debe ser clara y comprensible para una amplia variedad de lectores (jueces, técnicos de servicios sociales y los propios padres). Por todo ello, Budd (2005) recomienda que se tengan en cuenta los siguientes aspectos:

- Debe proporcionar una cronología y detalle de las actividades de evaluación realizadas (entrevistas, observaciones, pruebas psicológicas o de funcionamiento familiar, informes de otros recursos o servicios), las fechas de realización de dichas actividades y el nombre completo de las personas evaluadas.

- Si se utilizan categorías clínicas habría que explicarlas con lenguaje sencillo y fundamentar en qué se basa dicho diagnóstico. Es importante también destacar qué repercusiones tiene o podría tener sobre la vida familiar y laboral, indicando, por ejemplo, cómo incide sobre el ejercicio de la parentalidad o sobre las necesidades y el desarrollo de los hijos.

- Evitar hacer interpretaciones causales del tipo "el padre es incapaz de amar debido a su propia historia de maltrato" o predicciones sobre el futuro "el padre volverá a maltratar", ya que implican causalidades lineales y unidireccionales que no pueden ser fundamentadas o corroboradas.

- Evitar hacer recomendaciones sobre decisiones legales que están fuera de las competencias pro- 
fesionales. Por el contrario, hay que ofrecer descripciones factuales sobre la situación educativa en la familia, sobre recomendaciones de mejora de la misma, sobre necesidades específicas de los menores no cubiertas por sus padres, etc.

- Hacer alusión a las características del contexto donde se mueve la familia y las posibles oportunidades o limitaciones que éste representa para el desarrollo del menor.

No quisiéramos finalizar el presente artículo sin exponer las recomendaciones que realiza White (2005) para mejorar las evaluaciones sobre la capacidad parental. Según esta autora, además de adoptar un enfoque centrado en las fortalezas de la familia, es necesario garantizar al máximo la calidad de la evaluación y las decisiones que se derivaran de dicha evaluación. Para ello es necesario usar múltiples fuentes de información, evitar confiar excesivamente en instrumentos estandarizados que no se han diseñado específicamente para evaluar la parentalidad, reconocer la deseabilidad social en las respuestas de los padres, aceptar la responsabilidad y la disponibilidad para el cambio de los padres, implicar y hacer participes a los miembros de la familia en la evaluación realizada, prevenir los propios sesgos del profesional y adaptar la evaluación a las características de los padres, los hijos y el contexto en el que se desarrolla la vida familiar. En suma, el enfoque centrado en las competencias parentales implica un punto y aparte en la forma habitual de evaluar e intervenir con las familias en situación de riesgo psicosocial que sólo ahora estamos empezando a vislumbrar.

\section{Referencias}

Azar, S. T. (1998). Parenting and child maltreatment. En I. E. Sigel and K. A. Renninger (Volume 4. Eds.), Child Psychology in practice (pp. 361388). Handbook of Child Psychology. (5 $5^{\text {th }}$ edition) (W. Damon, Ed.). New York: John Wiley y Sons.

Azar, S. T., Cote, L. R. (2002). Sociocultural issues in the evaluation of the needs of children in custody decision making: What do our current frameworks for evaluating parenting practices have to offer? International Journal of Law and Psychiatry, 25(3), 193-217.

Azar, S. T. y Weinzierl, K. M. (2005). Child Maltreatment and Childhood Injury Research: A Cognitive Behavioral Approach. Journal of Pediatric Psychology, 30, 598-614.

Belsky, J., y Jaffee, S. R. (2006). The multiple determinants of parenting. En Cicchetti, D., y Cohen, D. J. (Eds.), Developmental psychopathology, Vol 3: Risk, disorder, and adaptation (2nd ed.) (pp. 38-85). Hoboken, N. J, US: John Wiley y Sons.

Budd, K.S. (2005). Assessing parenting capacity in a child welfare context. Children and Youth Services Review, 27(4), 429-444.

Cauce, A. M., Stewart, A., Rodriguez, M. D., Cochran, B. y Ginzler, J. (2003). Overcoming the odds? Adolescent Development in the context of urban poverty. En Luthar (Ed.) Resilience and Vulnerability. Adaptation in the context of childhood adversities. Cambridge University Press. Nueva York.

Díaz, A., Pérez, J., Martínez, M. T., Brito, A., y Herrera, E. (2001). El vínculo de apego en bebés prematuros: incubadora y método canguro. Revista de Atención Temprana, 4 (1), 11-19.

Gordon, L., Bradley, J., Aue, N., y Holman, A. (2006). Building on family strengths: Research and services in support of children and their families. 2005 conference proceedings. Portland, OR: Portland State University, Research and Training Center on Family Support and Children's Mental Health.

Hawkins, J. D., Catalano, R. F., y Miller, J. Y. (1992). Risk and protective factors for alcohol and other drug problems in adolescence and early adulthood: Implications for substance abuse prevention. Psychological Bulletin, 112(1), 64-105.

Kalil, A. (2003) Family Resilience and Good Child Outcomes. A review of the literature. Center for Social Research and Evaluation, Ministry of Social Development. Nueva Zelanda. www.msd. govt.nz/documents/publications/csre/family-resilience-good-child-outcomes.pdf

Luthar, S. S. (2003) Resilience and vulnerability. Adaptation in the context of childhood adversities. Cambridge University Press. Nueva York.

Máiquez, M.L., Rodrigo, M.J., Capote, C. y Vermaes, I. (2000). Aprender en la vida cotidia- 
na. Un programa experiencial para padres. Madrid: Visor.

Masten, A. S., y Curtis, W. J. (2000). Integrating competence and psychopathology: Pathways toward a comprehensive science of adaption in development. Development and Psychopathology, 12(3), 529-550.

Reder, P., y Duncan, S. (2003). Understanding communication in child protection networks. Child Abuse Review, 12(2), pp. 82-100.

Reder, P.; Duncan, S. y Lucey, C. (2003) Studies in the assessment of parenting. Routlegde. Nueva York.

Rodrigo M. J. y Palacios, J. (1998). Familia y desarrollo humano. Madrid. Alianza Editorial.

Rodrigo, M. J.; Máiquez, M. L.; Martín, J. C. y Byrne, S. (2008). Preservación Familiar: un enfoque positivo para la intervención con familias. Madrid: Pirámide.

Rodríguez, G., Camacho, J., Rodrigo, M. J., Martín, J. C. y Máiquez, M. L. (2006). La evaluación del riesgo psicosocial en las familias canarias usuar- ias de los servicios sociales municipales. Psicothema, 18(2), 200-206.

Simón, M. I., Correa, N., Rodrigo, M. J., y Rodríguez, M. A. (1998). Desarrollo y educación familiar en niños con cursos evolutivos diferentes. En M. J. Rodrigo y J. Palacios (Eds.), Familia y Desarrollo humano (445-464). Madrid: Alianza Editorial.

Tomison, A.M. (1999). Ensuring the protection of children: the role of child protection services in the identification, assessment ant treatment of maltreated children, Keynote presentation, Entry Parameters to Child and Family Services Workshop, NSW Department of Community Services. Australia.

Waters, E. y Sroufe, L.A. (1983). Social competence as a developmental construct. Developmental Review, 3, 79-97.

White, A. (2005). Assessment of parenting capacity. Literature review. Centre for Parenting and Research. Ashfield. Australia: Department of Community Services.

Manuscrito recibido: 02/11/2009

Revisión recibida: 17/11/2009

Manuscrito aceptado: 19/11/2009 